\title{
Comparison of Energy Consumption of an Optimized Gait Cycle between Human-like and Bird-like Leg Models
}

\author{
Rodrigo Matos Carnier \\ Graduate School of Engineering \\ Yokohama National University \\ Yokohama, Kanagawa 240-8501 \\ Email: rodrigo.carnier@gmail.com
}

\author{
Yasutaka Fujimoto \\ Graduate School of Engineering \\ Yokohama National University \\ Yokohama, Kanagawa 240-8501 \\ Email: fujimoto@ynu.ac.jp
}

\begin{abstract}
This work proposes to compare the energetic efficiency of two models of leg: human-like model, with leg bent backward, and bird-like mode, with leg bent forward. The models are represented by a manipulator with 2 degrees of freedom, which simulates the two phases of gait: swing phase (leg swinging) and support phase (foot in the ground, supporting the dislocation of the hip forward). After generating the gait trajectory and simulating the swing and support phases separately in the multibody simulator SimMechanics of the software Matlab, an energetic analysis is performed calculating the energy consumption through the power applied on the joints.

In order to focus the performance comparison on the physical dynamics of the models and eliminate inneficiency from the trajectory generation itself, the control solution is generated using a pseudospectral method of optimal control implemented in the software DIDO. The results point to the advantage of the human morphology in bending more freely in lift-off, causing the leg to use better the gravity to swing, and in making use of the increase of potential energy in swing phase to slow down the leg until heel strike.
\end{abstract}

\section{INTRODUCTION}

Robotic bipedal walking is a relatively recent field of research of engineering that was inspired by the human bipedal locomotion. It aims to develop robots that can perform tasks like humans do, in order to help or substitute humans at such tasks. Walking is one of the most important abilities expected from bipedal robots. However, there is still a number of performance issues to be solved or improved before robots that walk can substitute humans in daily tasks. One of the issues is the reduction of energy consumption in order to extend the duration of autonomous work.

The majority of bipedal robot prototypes created so far were inspired in the human morphology. However, if more inspiration can be drawn from nature, there is a second biological model: birds. The differences between human and bird walking start at the behavior of the trunk: in humans, the trunk stays erect during walk, while a bird trunk stays bent or crouched. This difference impacts the role and behavior of the segments of leg in a number of ways. The anatomy of legs of both humans and birds has three main segments: the thigh, the shin and the foot. However, in humans the thighs have a propelling function, while in birds they stay mostly in the horizontal position and have a balancing function. Human feet have its ankle almost at ground level, and a big sole in contact with the ground that supports most of the weight of the body; bird feet have its ankle high above the ground, at an height equivalent to the human knee's height, and do not have much of the body weight supported by the base of foot. Human toes are small in comparison to the size of the foot sole and have mainly a balancing function; bird toes are long and extend directly from the part of the feet contacting the ground, supporting a significant part the weight of the body. But even though each of these characteristics impact deeply the kinematics and dynamics of bird and human walking, another morphological difference possibly has the biggest impact in the dynamics of locomotion: the dynamics of the middle joint in the leg. Human legs bend backward through the knee joint, while bird legs bend forward through the ankle joint.

The study of energetics of bipedal walking is an old field in Zoology, and many studies were dedicated to it in the past [3] [4]. Human walking presents one of the most economic gaits between animals, but some types of bird (the wading birds) were reported to possess a better Metabolic Cost of Transport than human beings [5] [6]. Both morphologies are valid alternatives for energetically economic robotic bipedal walking.

In the field of engineering, studies recently started investigating bird-walking and/or comparing it with humanwalking for application in biped robotics. Abourachid and Hugel [1] compared the kinematics and dynamics of a quaillike and a human-like bipedal model as inspiration for robotics, and showed that a quail-like walker has better stability and flexibility in changing stride length without getting out of course. Hubicki [2] investigated the highly dynamic walking of running birds, like ostriches and quails, and developed an optimized gait for human-like prototypes, based on the control of a spring-mass model inspired in the walking characteristics of birds. However, no study so far focused on investigating the differences between the morphology of humans and birds as a design factor for improving the energy consumption of a 
robot model.

This paper investigates the impact of the bending behavior of a leg in the energetic efficiency of a robotic model of leg. A comparison of the energy consumption performance of two simple leg models (a human-like and a bird-like model) is presented. Both legs are modeled as a 2 degree of freedom manipulator rotating in the sagittal plane (the vertical plane XZ). The movement of a single leg is studied for both phases of gait cycle: swing phase and support phase. The trajectory generation is achieved by optimal control, changing the angular trajectory and velocity of links from an initial state to a final state, while minimizing the torque applied on joints and the angular velocity of joints. In this way, the control side of the study is guaranteed to be optimized, in order to limit the comparison of performance between both leg models to the differences in the dynamics of the mechanical systems.

\section{FORMULATION OF PROBLEM}

In this work, both the human leg and the bird leg are modeled as a manipulator of 2 degrees of freedom. The human and the bird gaits are studied by dividing the movement of a single leg in two phases: 1) swing phase, where the model is fixed at the hip joint and the end-effector (foot) is free; and 2) support phase, where the model is fixed at the foot (which becomes a fixed joint) and the hip becomes the free end-effector. The trajectory generation is performed optimizing the rotation of the two joints from an initial state to a final state in order to apply the least torques possible and have the least angular velocities possible. The optimal control method utilized is a pseudospectral method based on the Pontryagin's Minimum Principle and the Covector Mapping Theorem, developed by Ross [10] and implemented in the commercial numerical solver DIDO, used in this study. After generating the optimal inputs, the system is controlled in open loop. The two phases are generated as two different tasks, and simulated separately. After the simulation, an energetic study is performed over the results.

\section{A. System dynamics}

In order to obtain a more concise formulation and improve the computational efficiency of the numerical solver in later stages of the problem formulation and its solution, the system states are defined in terms of the absolute angular position of the links (the angle $\theta$ and the angular velocity $\omega$ of the links in respect to the lateral axis Y). The figure 1 shows the planar model of leg used in simulations, with the absolute reference for the states.

The dynamics of the mechanical system is derived form the Euler-Lagrange equations of the system, resulting in equation 1:

$$
M \ddot{\theta}+B=\eta
$$

Where $M$ is the Total Inertia Matrix, $\ddot{\theta}$ is the angular acceleration of the links, $B$ is the Vector of Coriolis and Gravitational Effects and $\eta$ is the torque applied on links.

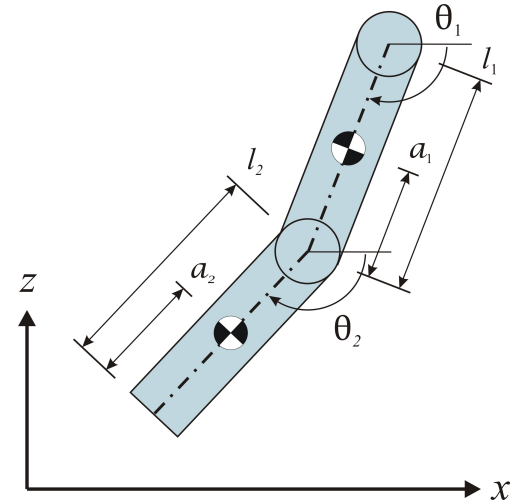

Fig. 1. Swing phase model of leg, modeled as a 2 dof manipulator fixed at the hip joint.

To account for energetic losses due to friction in joints, a friction term $\mu \dot{q}$ is added to the left size of the equation, where $\mu$ is the friction coefficient (value taken as 0.01) and $\dot{q}$ is the vector of angular velocities of joints. A transformation from angular velocities in joint reference $(\dot{q})$ to angular velocities in link reference $(\omega)$ is necessary to maintain all variables in the same reference, and is performed through the transformation matrix $K^{-1}$. The definition of the matrix will be given later in the paper.

$$
M \ddot{\theta}+B+\mu\left(K^{-1} \omega\right)=\eta
$$

If we group the states of the system,vector of angle of links $\theta$ and vector of angular velocity of links $\omega$, in a vector $x(t)$ of double their size:

$$
x(t)=\left[\begin{array}{c}
\theta \\
\omega
\end{array}\right]=\left(\theta_{1}, \theta_{2}, \dot{\theta_{1}}, \dot{\theta_{2}}\right)^{T}
$$

We obtain the following formulation of the system dynamics in state-space:

$$
f(x(t), u(t))=\dot{x}(t)=\left[\begin{array}{c}
\omega \\
M^{-1}\left(\eta-B-\mu\left(K^{-1} \omega\right)\right)
\end{array}\right]
$$

Since the task of walking has contact interactions with the floor, the dynamics of the environment needs to be accounted for. The unilateral contact forces applied by the floor on the foot of the model can be modeled as an inequality constraint on the end-effector position (the direct kinematics expression of the manipulator [9]). When applied in the solution method of the trajectory generation, this constraint guarantees that the foot is never set to crosses the floor line and go underground:

$$
\begin{gathered}
g(\theta)=l_{1} \sin \left(\theta_{1}\right)+l_{2} \sin \left(\theta_{2}\right) \\
g(\theta)>=0
\end{gathered}
$$




\section{B. Optimal Trajectory Generation}

The optimal control method utilized to generate the optimized leg trajectory is a pseudospectral method based on based on the Pontryagin's Minimum Principle and the Covector Mapping Theorem [10]. The method relies on discretizing the system and optimizing the trajectory of states by means of the minimization of the control Hamiltonian of the system. The optimization of the performance of the system is first defined in an Objective Function that describes mathematically what is being minimized. Then, by application of the Conditions of Optimality presented by Pontryagin's Principle, the system states are dualized into the costates, which are functions that "weights" the rate of change of states (its derivatives). The relationship between the optimal states, the optimal costates and the control Hamiltonian guarantees that the Hamiltonian achieves its minimum value over all the trajectory only when the states and costates are optimal. DIDO's pseudospectral method, created in beginning of the 2000's, solves some historical conflicts between direct and indirect methods of optimization and presents a very computationally efficient and precise optimal control method. It avoids the hardship of solving a Boundary Value Problem, and instead discretizes the system problem first and then performs a curve fitting that satisfies at the same time the trajectory generation, the Hamiltonian minimization and the Conditions of Optimality. For a more in-depth explanation of Pontryagin's Minimum Principle and its application to robotics refer to [7] [8]. For more details on DIDO and its pseudospectral method, refer to [10].

\section{Formulation of optimal problem}

Independetly of the optimal control method used, the formulation of an optimal control problem always starts with the definition of the performance criterion that is being minimized or maximized. This criterion is the objective function:

$$
J\left(x(t), u(t), t_{f}\right)=h\left(x\left(t_{f}\right), t_{f}\right)+\int_{t_{0}}^{t_{f}} l(x(t), u(t)) d t
$$

Where $h\left(x\left(t_{f}\right), t_{f}\right)$ is the Terminal Cost (or Mayer Cost), an end-point constraint for the state of the system, and $l(x(t), u(t))$ is the Running Cost (or Lagrange Cost), the performance criterion for optimality of the solution, subjected to the dynamics of the system. In this work, the objective is the minimization of energy consumption of a leg model. Since the actual power applied by the joints are the product of torque and angular velocity of the link, both terms are defined in the function. However, defining this product as the Running Cost makes it difficult to find a solution, since the resulting optimization problem is not convex. To circumvent this, both terms are defined separately and squared inside the Running Cost.

The energy consumption criterion will be defined in terms of the square of torque applied on each joint of the manipulator. However, in order to achieve more concise analytical expressions, the coordinates system used is based on angles of links and torques applied on links, not on angles of joints and torques applied on joints. Thus, it is necessary to perform a transformation from angle of joints $(q)$ to angle of links $(\theta)$, expressed by equation 7 . In the same way, a transformation from torque applied on joints $(\tau)$ to torque applied on links $(\eta)$ is expressed by equation 8 . With these transformations, we can obtain the formulation of the Objective Function shown in equation 9:

$$
\begin{gathered}
q=K^{-1} \theta \\
K=\left[\begin{array}{ll}
1 & 0 \\
1 & 1
\end{array}\right] \\
\tau=G^{-1} \eta \\
G=\left[\begin{array}{cc}
1 & -1 \\
0 & 1
\end{array}\right] \\
J\left(x(t), u(t), t_{f}\right)=\int_{t_{0}}^{t_{f}}\left(\frac{a}{2}\left(G^{-1} \eta\right)^{T}\left(G^{-1} \eta\right)+\right. \\
\left.\frac{b}{2}\left(K^{-1} \omega\right)^{T}\left(K^{-1} \omega\right)\right) d t
\end{gathered}
$$

In this expression, the Terminal Cost is zero and the Running Cost consists of both the square of torques and square of angular velocities of joints. $a$ and $b$ are weighting factors.

After defining the Objective Function, the application of Pontryagin's Minimum Principle starts by deriving the control Hamiltonian, presented in equation 10 :

$$
\begin{aligned}
H= & \frac{a}{2}\left(G^{-1} \eta\right)^{T}\left(G^{-1} \eta\right)+\frac{b}{2}\left(K^{-1} \omega\right)^{T}\left(K^{-1} \omega\right)+ \\
& \lambda_{\theta}^{T} \cdot \omega+\lambda_{\omega}^{T} \cdot\left(M^{-1}\left(\eta-B-\mu\left(K^{-1} \omega\right)\right)\right)
\end{aligned}
$$

Taking the formulation of the system dynamics, the floor constraints, the desired initial/final states of the system and the Objective Function for the given problem as an input parameters, the solver DIDO is then used to generate a candidate extremal solution for the problem. The solution is validated as an optimal solution checking for the conditions of optimality and for the feasibility of the solution in a multibody simulator.

\section{IMPLEMENTATION AND RESULTS}

After presenting the models of leg, the generated trajectory will be presented, followed by the energy consumption analys.

\section{A. Model}

The parameters of the 2 degrees of freedom manipulator are the same for both the human leg and the bird leg, and are presented in table I. The only difference is the bending of the knee: the human leg bends backward and the bird leg bends forward. The parameters were extracted from a human subject of an experiment where human walking kinematics were measured [12]. 


\begin{tabular}{|c|c|c|}
\hline LINK & LENGTH & MASS \\
\hline Link1 & $0.314 \mathrm{~m}$ & $5.67 \mathrm{~kg}$ \\
\hline Link2 & $0.425 \mathrm{~m}$ & $2.637 \mathrm{~kg}$ \\
\hline
\end{tabular}

DIMENSIONS AND MASS OF 2 DOF MANIPULATOR

\section{B. Task definition}

The task is the generation of an optimal trajectory for the link angles, in order to bring them from the initial state to the final state inside the window of time of 1 second, with initial and final velocities equal to zero. Initial and final postures have bent knees in order to keep the Center of Pressure (and the Zero-Moment Point) of the complete robot model (to be developed in the future) inside the support polygonon of the foot.

The task is the generation of an optimal trajectory for the link angles, in order to bring them from the initial state to the final state inside the window of time of 1 second, with initial and final velocities equal to zero. The initial and final states of the human leg model, for both swing phase and support phase are shown in Figure 2, while the states of the bird leg model, for both phases, are shown in Figure 3. The height of the hip is kept constantly, forcing the knee to be bent during the transition from initial to final states. This specification is intended to approximately reproduce common trajectory generation methods for bipedal locomotion based on the ZeroMoment Point [11], where the height of Center of Gravity is kept constant.
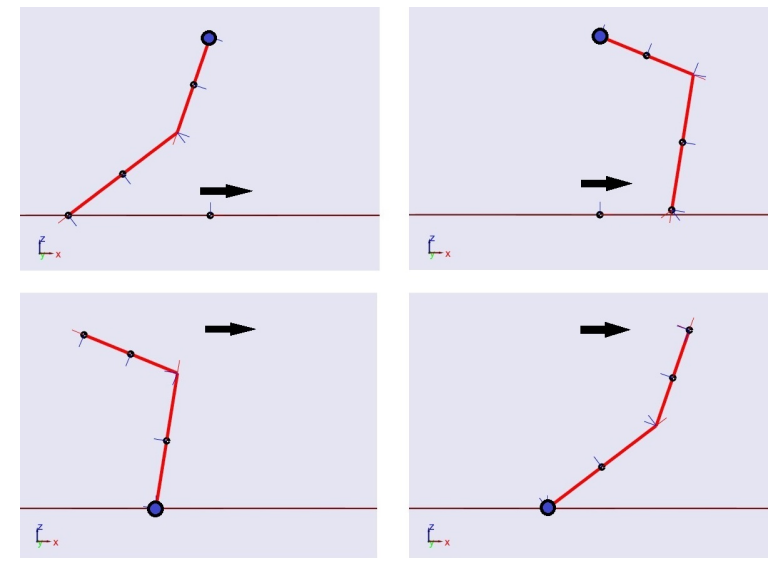

Fig. 2. The top figures are the initial and final state of the human leg model for the swing phase. The bottom figures are the states for the support phase.

\section{Validation}

The feasibility of both solutions was validated using the multibody simulator SimMechanics present in MATLAB/Simulink software. The leg models correctly moved from the initial conditions to the final conditions respecting the set time (1 second).

An important difference of behavior of the leg models was perceived in the video simulations. The human leg model first lifted the lower link then swinged both links with the help of
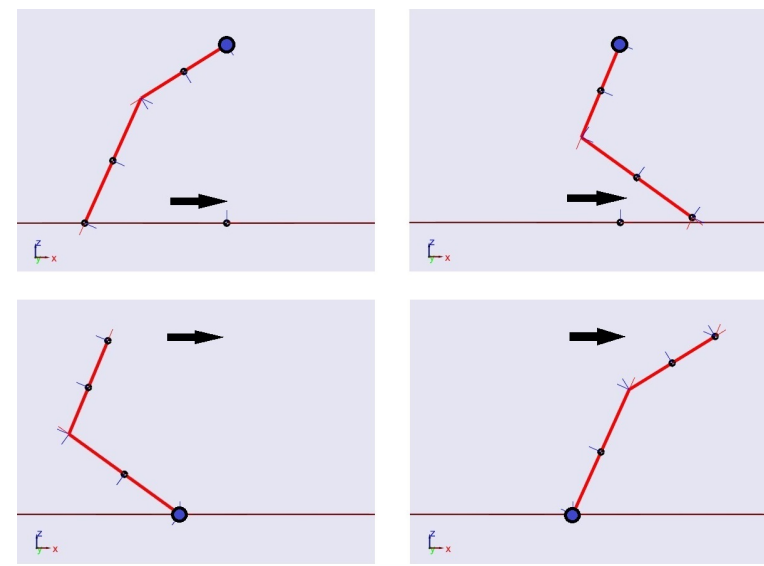

Fig. 3. The top figures are the initial and final state of the bird leg model for the swing phase. The bottom figures are the states for the support phase.

the gravitational pull. Due to the posture in the initial and final states, the desacceleration in the final state coincided with the increase of potential energy, and the movement showed a smoothness. The bird leg, though, had a decrease of potential energy alongside initial and final states, and the higher velocity of the links generated an overshoot of position. It is noticeable, however, that the optimization algorithm found it more efficient to generate a relative burst of velocity with the correction of the overshoot than to generate a slow movement without overshoot.

Figure 4 presents the trajectory of states and controls for the human leg model, while Figure 5 presents the same for the bird leg model. All figures of trajectory of states have the same scale, as well as the figures of trajectory of controls.
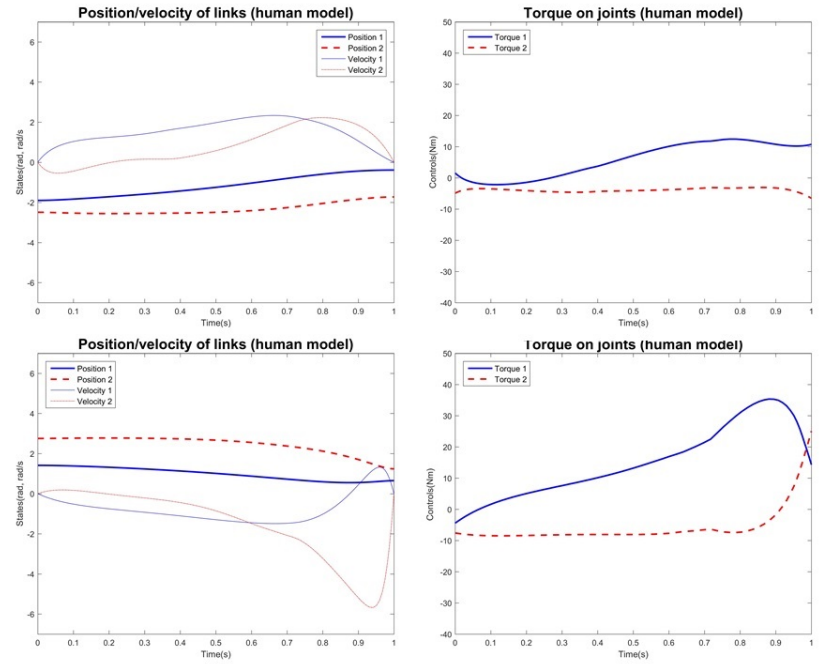

Fig. 4. Optimal solution trajectory of the human leg model. The top figures are the states (left) and control (right) trajectories of the swing phase. The bottom figures represents the support phase.

A first observation of the progression of states and controls indicates that the bird leg model requires bigger torque peaks to perform the same task. Velocities of bird leg links also 

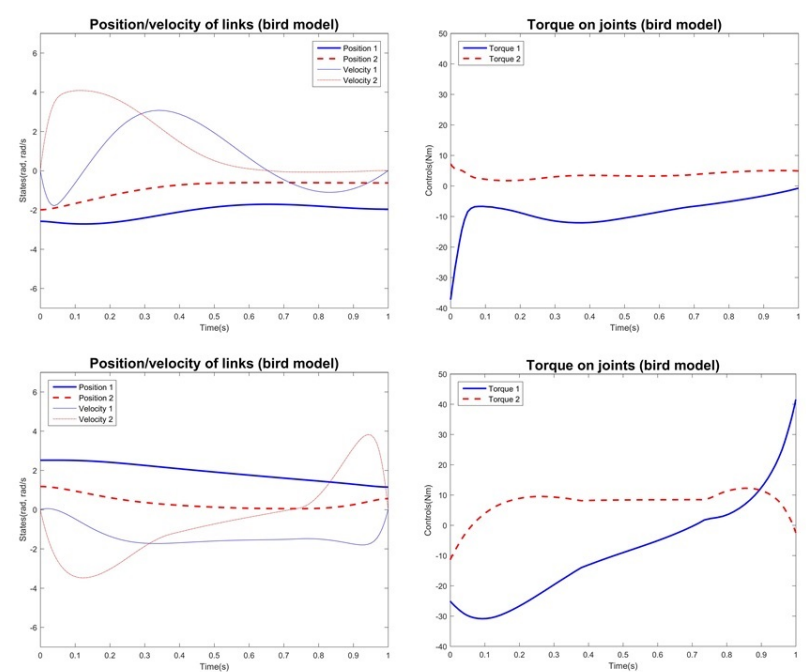

Fig. 5. Optimal solution trajectory of the bird leg model. The top figures are the states (left) and control (right) trajectories of the swing phase. The bottom figures represents the support phase.

vary more, indicating more accelerations and desaccelerations than presented in the human model. Further energetic analysis clarified the implications of this behavior.

\section{Energetic analysis}

Table II shows the value of the Objective Function (the integral of square of torques applied on joints and of square of angular velocities of joints) computed along the trajectory of the leg. The values are adimensional:

\begin{tabular}{|c|c|c|}
\hline MODEL & Obj. Func. Swing & Obj. Func. Support \\
\hline Human leg & 35.7 & 181.1 \\
\hline Bird leg & 36.2 & 203.6 \\
\hline \multicolumn{3}{|c|}{ TABLE II } \\
\hline
\end{tabular}

COMPARATION OF OBJECTIVE FUNCTION FOR HUMAN LEG AND BIRD LEG, IN SWING PHASE AND SUPPORT PHASE.

This table already shows how the bird leg model generates a bigger value of cost function to move the foot from the same initial state to the same final state as the human leg model. However, even though the minimization of the torques applied on the joints are a good approximation of the energy consumption of the system, a proper analysis needs to compare the actual power applied on the joints and its impact on the variation of mechanical energy in the system.

To begin this comparation, first Figures 6 and 7 are presented. The first shows a plot of the trajectory of power applied on joints, for both the human and the bird solutions, in the swing phase. The last shows this result in the support phase.

Table III shows two types of energy measurements: 1) the value of the variation of mechanical energy between initial and final states, for each model and each phase of gait, and 2) the value of the integral of power applied on the joints along the trajectory, for each model and each phase of gait.

In order to compare the effort of both controls, the variation of mechanical energy needs to be subtracted from the power

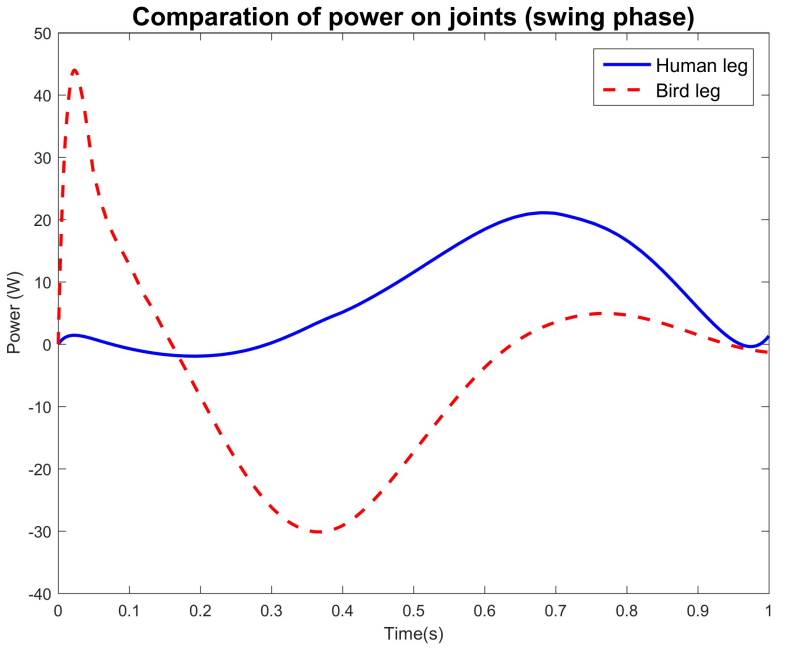

Fig. 6. Comparation of sum of power applied on all joints for human leg model and bird leg model in the swing phase.

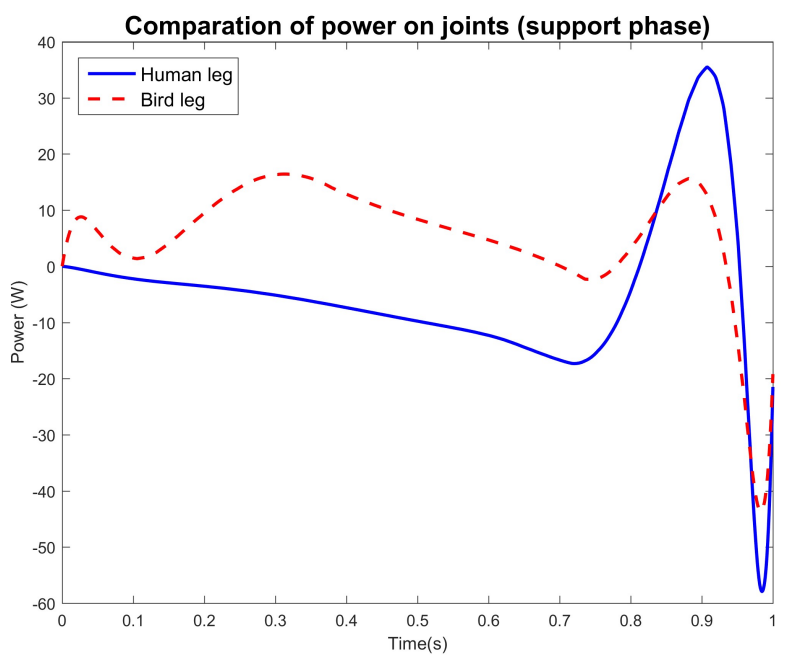

Fig. 7. Comparation of sum of power applied on all joints for human leg model and bird leg model in the support phase.

exerted on the joints. After this offset removal, the values of Table IV were obtained. The two types of information presented are: 1) the difference between the integral of power and the variation of Mechanical Energy for the whole gait cycle, for each model, and 2) the percentage of this difference in respect to the average mechanical energy along the whole gait cycle. This difference between power and mechanical energy is the actual expenditure of power, in terms of waste, since this surplus of power is not being converted into mechanical energy (potential, in case of boundary postures). The magnitude of this surplus in respect to the total mechanical energy gives an approximation of efficiency of conversion of power applied on the joints into change of mechanical energy, and is used here as the measure of energetic efficiency of each gait. 


\begin{tabular}{|c|c|c|c|c|}
\hline MODEL & Mec.En. & Int.Power & Mec.En. & Int.Power \\
\hline & \multicolumn{2}{|c|}{ Swing phase } & \multicolumn{2}{c|}{ Support phase } \\
\hline Human leg & $7.45 \mathrm{~J}$ & $7.64 \mathrm{~J}$ & $-5.11 \mathrm{~J}$ & $-5.02 \mathrm{~J}$ \\
\hline Bird leg & $-4.79 \mathrm{~J}$ & $-4.46 \mathrm{~J}$ & $5.32 \mathrm{~J}$ & $5.42 \mathrm{~J}$ \\
\hline
\end{tabular}

VARIATION OF MECHANICAL ENERGY AND OF THE INTEGRAL OF POWER BETWEEN HUMAN LEG AND BIRD LEG.

\begin{tabular}{|c|c|c|}
\hline MODEL & Power - Mec.Energy & \% of Power wasted \\
\hline Human leg & $0.283 \mathrm{~J}$ & $4.32 \%$ \\
\hline Bird leg & $0.418 \mathrm{~J}$ & $8.6 \%$ \\
\hline
\end{tabular}

TABLE IV

WASTED POWER APPLIED ON JOINTS AND PERCENTAGE TO AVERAGE MECHANICAL ENERGY.

\section{E. Discussion}

According to the quantitative analysis of the simulation data, the positive net energy (the waste of energy through friction) is higher in the bird model gait than in the human model gait (around $8.6 \%$ of total variation for the bird model against $4.32 \%$ for the human model), indicating that the optimization of movement of a human leg model is more efficient than of a bird leg model for the same task. It is important to clarify that this task has some particular parameters, assumptions and limitations: the parameters of the leg, a given size of the leg step, walking with a constant height of hip and the initial and final postures assumed to respect the ZMP balance criterion in a complete model with two legs.

Taking into consideration the behavior of legs during simulation, discussed in the subsection Validation, and the higher velocity peaks of the bird model, the proposed cause for the difference of efficiency between the two leg models is the behavior of the links in respect to their acceleration.

Analysing the Figures 4 and 5, together with the video of the simulation, it is noticeable how the velocity of the bird leg has higher peaks than the human leg velocity, but that it does not affect the total duration of the gait (1s for each of the two phases). Higher peaks of velocity generates more losses due to friction. Besides, the bird leg is not able to start swinging and to lift the lower link at the same time, since the initial posture of the bird leg locks the foot against the floor. This inability to swing the leg with the help of gravity are interpreted as generating wasted effort against it. Also, the overshoot of final position during the swing phase of the bird leg model is supposed to generate even more waste, due to two additional accelerations/desaccelerations of the leg. Due to the decrease of potential energy, a natural acceleration towards the final posture happens and makes the optimization process to prefer keeping the velocity of the links than working slowly against the gravity.

Given all the points above, the results indicate a worse posture constraint in the bird leg model that is incurred when the design assumptions of this simulation are assumed by a full robot prototype, like constant hip height and the balance strategy based on the Zero-Moment Point (semi-static).

\section{Summary AND Future Works}

This paper realized the optimization of energy consumption in the task of moving two leg models in the swing phase and the support phase of a biped step, utilizing pseudospectral method of optimal control based on Pontryagin's Minimum Principle and Covector Mapping Theorem. The first leg model was based on human bipedal walking, while the second leg model was based on bird bipedal walking. Losses by friction were included in the models. A comparation of energetic consumption was performed between the tasks of both models, indicating a possibility of the bird-like model possessing dynamics that produce a less energetically efficient locomotion given some common assumptions of robotic bipedal walking (constant hip height, bent knee and positioning of the Center of Gravity in order to keep the Center of Pressure inside the polygonon of support).

The next activities will be the deeper investigation of the constraints that generate a difference of energy consumption efficiency in the bird bipedal walking in respect to human bipedal walking, like modelling different postures (extending the knees, for example) and the application of a different balance strategy that does not require such assimetrical postures of the leg between initial and final states (like strategies based on the inverted pendulum model). Also, more complex models will be studied, with the insertion of more degrees of freedom and complexities in the models, to better represent the real dynamics of legs of humans and birds.

\section{REFERENCES}

[1] V. Hugel, A. Abourachid, "The Natural Bipeds, Birds and Humans: An Inspiration for Bipedal Robots", Biomimetic and Biohybrid Systems, pp.3-15, 10.1007/978-3-319-42417-0_1, Springer International Publishing, ISBN: 9783319424170, 2016.

[2] C.M. Hubick, "From Running Birds to Walking Robots: Optimization as a Unifying Framework for Dynamic Bipedal Locomotion", Ph.D Thesis, Oregon State University, 2014.

[3] S.M. Gatesy, A.A. Biewener, "Bipedal locomotion: effects of speed, size and limb posture in birds and humans", Journal of Zoology 224, 127147, 10.1111/j.1469-7998.1991.tb04794.x, 1991.

[4] G.A. Cavagna, M. Kaneko, "Mechanical work and efficiency in level walking and running", Journal of Physiology, 268:467-48 I, 1977.

[5] R.M. Alexander, "Bipedal animals, and their differences from humans", J Anatomy, 204(5), pp.321-330, 2004.

[6] L.W. Bruinzeel, T. Piersma, M. Kersten M.F. and Leopold,"Low costs of terrestrial locomotion in waders", Ardea, 87(2), 199-205, 1999.

[7] D.E. Kirk, "Optimal Control Theory: An Introduction", Dover Publications, ISBN: 9780486434841, Mineola, New York, 2004.

[8] R. Callies and P. Rentrop, "Optimal Control of Rigid Link Manipulators by Indirect Methods". GAMM-Mitteilungen, vol. 31, no. 1, pp. 27-58, 2008.

[9] B. Siciliano and O. Khatib, "Springer Handbook of Robotics", Springer, Berlin, 2008.

[10] I.M. Ross, "A Primer on Pontryagin's Principle in Optimal Control", Collegiate Publishers, ISBN: 9780984357109, 2009.

[11] M. Vukobratovic and B. Borovac. "Zero-moment Point - Thirty Five Years of its Life". International Journal of Humanoid Robotics Vol. 1, No. 1, pp. 157-173, Institute Mihajlo Pupin, Serbia and Montenegro, 2004.

[12] D.A. Winter, "Biomechanics and Motor Control of Human Movement", Second Edition, A Wiley-Interscience Publication, Ontario, Canada, 1990. 